\title{
PERCEPTIONS OF COOPERATION IN A LONGITUDINAL SOCIAL DILEMMA
}

\author{
KELLY BOUAS HENRY \\ University of Oklahoma
}

\begin{abstract}
Most social dilemma studies of communication's effects on cooperation are based on laboratory results with trivial incentives. Studies of real-life social dilemmas with nontrivial rewards are needed to extend the generality of laboratory results. Perceptions of cooperation on a group product (weekly group essay) were examined as a public goods dilemma embedded in a longitudinal study of groups using either face-to-face or computer-mediated communication. Perceptions of cooperation increased over time, whereas measures of group identity did not. No media effect on perceptions of cooperation was observed. Several predictors of late perceptions of cooperation were examined, but only early perception of cooperation was significant. Results are discussed with respect to several current hypotheses regarding communication and cooperation in social dilemmas.
\end{abstract}

Much of the study of interpersonal cooperation has been done in the context of social dilemmas. These are situations in which each group member receives a higher pay-off for a noncooperative choice (defection) than a cooperative choice no matter what other members do, but all members are better off if all cooperate than if all defect (Dawes, 1980; van Lange, Liebrand, Messick, \& Wilke, 1992). One robust finding from that work is that cooperation increases in groups who discuss the dilemma face-to-face before

AUTHOR'S NOTE: The research on which this article is based was supported in part by National Science Foundation grant IRI93-10099 (J. E. McGrath, principal investigator). I thank Holly Arrow, Jennifer L. Berdahl, Linda Lebie, Joseph E. McGrath, Kathleen O'Connor, and Jonathan A. Rhoades, who planned and conducted the workshop study on which this article is based. I also thank Martha Orland and others involved in data collection and processing. I thank Norbert L. Kerr, Samuel S. Komorita, Joseph E. McGrath, Kathleen M. O'Connor, and two anonymous reviewers for comments on earlier drafts. An earlier version of this article was presented at the 1995 meeting of the Midwestern Psychological

SMALL GROUP RESEARCH, Vol. 31 No. 5, October 2000 507-527

(c) 2000 Sage Publications, Inc. 
making individual choices (Komorita \& Parks, 1995; Messick \& Brewer, 1983). Research to understand that relation has centered on two main hypotheses: (a) that increased cooperation following discussion arises because of an increase in group identity (Brewer \& Kramer, 1986) and (b) that increased cooperation following discussion arises because of commitments to cooperate made during discussion (Kerr, Garst, Lewandowski, \& Harris, 1997; Orbell, van de Kragt, \& Dawes, 1988).

Recent research suggests that commitments to cooperate may be the primary factor underlying increased cooperation after discussion. However, the bulk of that research-showing discussion's impact or testing either of the hypothesized causes - has been done with social dilemmas created in laboratories. Such situations differ in at least four important ways from real-life social dilemmas. Those differences, discussed extensively next, are such that they raise doubts as to the generality of the findings that commitment (not group identity) may be responsible for discussion's effect. This study examines social dilemmas in more realistic conditions and thereby tests the generality of findings about discussion-induced cooperation.

\section{DIFFERENCES BETWEEN LABORATORY} AND REAL-LIFE SOCIAL DILEMMAS

Whereas numerous differences exist between laboratory and real-life social dilemmas, four main distinctions are relevant to the present study. First, the incentive to cooperate or defect in laboratory social dilemma investigations of the discussion effect (Orbell et al., 1988) may be very weak. Participants often play for very small economic incentives (Ostrom \& Walker, 1991) and in some

Association, Chicago. A set of seven articles analyzing different data from the same longitudinal study was published as a special issue of Computer Supported Cooperative Work (Vol. 4, 1996). None of the analyses reported here have been previously published elsewhere. Analyses that might be mistaken for those previously published are specifically marked and explained in a footnote. Correspondence concerning this article should be addressed to Kelly Bouas Henry, who is now at the Department of Psychology, Missouri Western State College, 4525 Downs Dr., St. Joseph, MO 64507; e-mail: henry@griffon.mwsc.edu. 
cases even for lottery tickets rather than actual money (Bouas \& Komorita, 1996). Although laboratory situations capture the essence of the dilemma, participants in such situations may not be economically self-motivated to the degree they might be in realworld social dilemmas and thus are more responsive to experimental manipulations.

A second difference between laboratory and real-life social dilemma studies of the discussion effect has to do with communication. In laboratory situations, communication is controlled. Participants are told what topic to discuss, how long to discuss it, and what communication medium to use. In real-life social dilemmas, communication is almost always less constrained. People in more realistic dilemmas may discuss any topic using any available communication medium (e.g., e-mail and online computer-mediated communication). Because nearly all research on discussion-induced cooperation to date has been conducted in laboratory settings, it is reasonable to ask if results from those studies will generalize to more realistic settings. In addition, research on the effect of communication medium in a social dilemma is almost nonexistent (for an exception, see Kiesler, Sproull, \& Waters, 1996) and deserves attention as well.

A third major difference between laboratory and real-life social dilemma investigations is the meaning of time. Several social dilemma experiments have used one-shot choice designs to investigate a manipulation's impact on cooperation (Braver \& Wilson, 1986). Other social dilemma experiments require participants to make a series of choices (Kerr \& Kaufman-Gilliland, 1994). Regardless of whether a single choice or choices over trial blocks are analyzed, most social dilemma experiments are conducted within the context of the experimental hour. Laboratory results suggest that cooperation decreases over time (Isaac \& Walker, 1988; Komorita, Parks, \& Hulbert, 1992; Rapoport \& Chammah, 1965). In real life, social dilemmas typically unfold over much longer periods. For example, a team evaluated and rewarded based on performance on a group product is essentially experiencing a real-life public goods dilemma that continues for however long the team remains intact, a time period that could involve years of interdepen- 
dence. Studying real-life social dilemmas requires considering time more broadly.

Finally, the fourth difference between laboratory and real-life social dilemmas involves what is meant by cooperation. Cooperation has a specific meaning determined by the experimental task used in laboratory social dilemmas. In such situations, nothing is subjective about determining whether a group member has cooperated. Once the choices are made, one knows who has cooperated. Yet, real-life social dilemmas rarely have such clear-cut distinctions between cooperation and defection. Rather, people experiencing real-life dilemmas-such as the organizational team described earlier-rely on perceptions of cooperation to guide their behavior. Cooperation may be objective in the laboratory, but in real-life dilemmas, it is experienced subjectively. It is clear that expectations of cooperation do influence choice in laboratory social dilemmas (Bouas \& Komorita, 1996), a finding that suggests perceptions of cooperation should influence the development of cooperation in longer term, real-world social dilemmas.

Given these four differences, it is possible that experimental results relevant to communication in social dilemmas may not generalize to more realistic settings. The primary conclusions of the literature regarding discussion-induced cooperation are discussed in the context of real-life social dilemmas next.

\section{LABORATORY RESULTS AND THE QUESTION OF GENERALITY}

The group identity explanation. Conceptualizations of group identity have been based in social identity (Tajfel \& Turner, 1979) and social categorization (Turner, 1985) theories. One of social identity's main tenets is a positive relationship between group identity and intergroup differentiation (Hogg \& Abrams, 1988; Kelly, 1988). This relationship is suggested to cause in-group bias (Brewer, 1979; Rabbie, 1982). Research that supports this relationship has shifted the emphasis of in-group bias from negative consequences of out-group perceptions to positive consequences of group identity and hence higher levels of in-group cooperation over time. 
Group identity is hypothesized to cause group members to be more concerned with the outcomes of their fellow group members than their own outcomes (Kerr \& Kaufman-Gilliland, 1994). Once identification is evoked, cooperative behavior should follow because of the reordering of priorities. In an early study of discussion's effects, Dawes, McTavish, and Shaklee (1977) compared four conditions varying in type of communication: (a) no communication, (b) group discussion of an irrelevant issue, (c) group discussion on the dilemma, and (d) group discussion on the dilemma plus nonbinding roll call of intentions. Decisions on the dilemma were made anonymously, and results showed a large difference in cooperation rates for Conditions B and C (35\% and 74\%, respectively). Dawes et al.'s explanation for this result suggests that only discussion of the dilemma evoked group identity and that group identity made members choose to cooperate. Unfortunately, the study did not provide an independent measure of group identity, and conclusions regarding its role in discussion-induced cooperation are tentative.

Brewer and Kramer (1986) and Kramer and Brewer (1984) argued that group identity occurs if members believe they are all recipients of a common fate rather than if they experience events individually. Brewer and Kramer (1986) manipulated common fate by leading participants to believe a single lottery would determine the amount that all would be paid per point earned in a social dilemma situation. Thus, all participants shared the same fate. Results of both studies indicated common fate groups reported higher group identity than control groups and individuals behaved more cooperatively when a collective group identity was salient.

Both Dawes et al. (1977) and Brewer and Kramer's (1986; Kramer \& Brewer, 1984) work investigated the group identity explanation without testing it against the commitment explanation. Later laboratory studies focused more on testing the group identity against the commitment explanation of discussion-induced cooperation.

The commitment explanation. In an extension of Dawes et al.'s (1977) work, van de Kragt, Orbell, and Dawes (1983) allowed some 
participants to discuss a public goods dilemma prior to making their choice, whereas others were not allowed to communicate. Participants discussing the dilemma designated a minimal contributing set so the public good could be provided at minimum cost. Van de Kragt et al. hypothesized that a social contract is defined during discussion, causing group members to feel obliged to adhere to designated roles. Consistent with this explanation, Orbell et al. (1988) found that commitments to cooperate made during discussion increased cooperation only when all group members promised to cooperate. Other research (Braver \& Wilson, 1986; Chen, 1996; Chen \& Komorita, 1994) also supports the commitment explanation.

Compelling evidence supporting a commitment explanation came from Kerr and Kaufman-Gilliland (1994) in a critical test of the group identity and commitment explanations. Kerr and Kaufman-Gilliland argued that if the group identity explanation is correct, collective welfare should be more important to the individual and a nonefficacious cooperative act is unlikely as it will not enhance collective welfare. Alternatively, if the commitment explanation is correct, the efficacy of the act should not matter. As expected based on the commitment explanation, participants in a public goods dilemma made cooperative choices even in lowefficacy conditions. In addition, an independent measure of group identity (Hinkle, Taylor, Fox-Cardamone, \& Crook, 1989) allowed the authors to remove group identity in a covariance analysis, yet the discussion effect remained.

Finally, Bouas and Komorita (1996) used a design similar to Dawes et al. (1977) but included the Hinkle et al. (1989) measure of group identity. In a competitive test of the group identity and commitment explanations, Bouas and Komorita found that cooperation increased only in conditions in which the dilemma was discussed (the only conditions in which commitments to cooperate are possible). Group identity was high in conditions where any face-to-face discussion (on the dilemma or not) occurred. In a series of regression analyses, Bouas and Komorita found the expectation that other group members would cooperate was the most important factor in explaining the variance in choice behavior. Assuming this expectancy arose from commitments made during group discussion of 
the dilemma, this result supports the commitment explanation and contradicts Dawes et al.'s earlier conclusion that only discussion of the dilemma evokes group identity.

In sum, evidence suggests group identity could contribute to discussion's influence on cooperation; however, the studies employing competitive tests of both explanations suggest the commitment explanation is the dominant mechanism underlying the discussion effect. Whether this conclusion generalizes beyond laboratory settings requires further empirical work.

Generalizing to more real-life social dilemmas. Does commitment increase cooperation in more real-life social dilemma settings? Or, is group identity a reasonable explanation in those contexts? In real groups, strong interpersonal bonds that may be associated with group identity should take time to develop (Hill \& Gruner, 1973; Maples, 1988; Tuckman \& Jensen, 1977). Real group members (rather than ad hoc groups in laboratory studies) should experience a much stronger version of group identity than is possible in any one-shot laboratory setting. The group identity explanation may be more likely to find support in real-life social dilemma settings than in laboratory ones. If it does not, we would have even stronger evidence that group identity is not the driving force behind discussion-induced cooperation.

The impact of communication media on cooperation should also be of interest in real-world social dilemmas. The communication medium that group members use may either inhibit or foster the development of group identity. Because computer-mediated communication (CMC) lacks the rich interpersonal cues present in face-to-face (FTF) interaction (Daft \& Lengel, 1986), CMC groups may not experience group identity to the extent FTF groups do. If group identity is inhibited by use of CMC and if group identity is an important mechanism underlying cooperation in natural groups, then $\mathrm{CMC}$ groups may report less cooperation than groups working FTF. If a group identity is not critical for cooperation in natural groups - as the commitment explanation argues-then a finding of no media differences in cooperation would bolster existing literature that supports the commitment explanation. 
Few studies have examined the impact of communication medium on choice in social dilemmas. Although some research demonstrated medium effects in mixed-motive tasks generally (O’Connor, Gruenfeld, \& McGrath, 1993), Kiesler et al. (1996) found participants in a laboratory social dilemma cooperated equally with a text-only computer and a confederate but less with a humanlike computer. Given the similarity of a text-only computer to the experience of synchronous $\mathrm{CMC}$, it may be reasonable to expect no effect of medium on cooperation.

Other laboratory results would be of interest to examine in more real-life social dilemma situations. One previously mentioned finding was that cooperation in laboratory social dilemmas almost always decreases over time. In more realistic longitudinal social dilemmas, should this effect replicate? If group identity is a viable mechanism behind cooperation in real-world social dilemmas, then it may well be that if group identity invariably develops with longer time periods experienced by natural groups, corresponding increases in cooperation may be observed.

\section{OVERVIEW}

The earlier discussion outlines several distinctions between laboratory and real-life social dilemma situations, focusing on research addressing the discussion effect. The differences point to ways in which laboratory research may not easily generalize to more realistic social dilemmas. The present study constitutes a first step toward examining these issues outside of a laboratory setting. The study is explicitly exploratory in nature given the paucity of research that focuses on generalizing laboratory social dilemma studies of the discussion effect to other contexts. However, two competing hypotheses based on the previous discussion will guide the analyses:

Hypothesis 1: If social dilemma findings are correct, then early levels of group identity should be unrelated to later measures of cooperation, but early expectations of cooperation should predict later measures of cooperation. Furthermore, no difference in cooperation or group identity should be observed in FTF versus CMC groups. 
Hypothesis 2: If group identity is a more viable explanation of communication's effects in real-life social dilemma situations, group identity should increase over time and cooperation should also increase. FTF groups should experience greater group identity and, consequently, cooperation than groups using CMC groups.

\section{METHOD}

\section{PARTICIPANTS AND DESIGN}

One hundred nineteen undergraduates enrolled in an organizational psychology course participated. Participants actively applied text and lecture material in a required weekly workshop in which the study took place. The present study employed a 2 (computermediated vs. face-to-face communication) $\times 2$ (early: Weeks 2 through 4 vs. late: Weeks 5 through 7) design with repeated measures on the second factor. Week 1 was not included inasmuch as a public goods dilemma did not occur that week.

\section{PROCEDURE}

Participants met in three- or four-person groups once a week for 7 weeks in a 2-hour workshop. Groups met in either FTF or CMC conditions. Group members worked on an individual task and then collaborated on a solution to the task they had worked on individually. The specific nature of the weekly tasks is described in McGrath and Arrow (1996), but all tasks required students to apply course material to an organizational consulting problem. After completing the task, participants completed a posttask questionnaire assessing feelings toward and perceptions of the group, the group's task performance, and the task itself.

Next, participants wrote individual essays relating the consulting task to the course material. Individual essays were always followed by a group essay in which participants collaborated on an identical assignment. After the group essay, participants filled out a postessay questionnaire assessing perceptions of contribution levels toward the group essay. Individual and group essay scores were 
part of participants' course grades. The only variant on this occurred during Week 1 in which no essays were written because there had not been enough course content presented for participants to complete the assignment. Groups were dissolved at the end of Week 7.

The group essay can be construed as a real-life public goods dilemma. It would be easy for a group member to withhold his or her contribution to the creation of the essay and free-ride to a good grade. However, if all members did this, then the group essay's quality would decline, leading to a poor grade, and all would be worse off than if all had contributed. In this study, cooperation was assessed by participants' perceptions of contribution to the group essay.

Measures of cooperation. Measures of contribution to group essays were taken from the postessay questionnaire. Participants rated their own and each other's amount of contribution to the group essay, the value of those contributions, the amount of effort put forth, and the number of ideas generated. The ratings of the first three measures were on a 7-point scale with 1 indicating the least amount of contribution, lowest value of contribution, and least amount of effort put forth and 7 indicating the most. The fourth rating (number of ideas) was also made on a 7-point scale, with 1 indicating no ideas and 7 indicating many. All ratings were made privately and anonymously on absolute rather than relative scales such that it would be possible for all members to receive ratings of 7 (highest rating).

To construct a cooperation measure for each week, participants' evaluations of themselves were removed and the remaining evaluations of each group member were averaged. This was done separately for each of the four measures of contribution. The four measures of contribution were then averaged to form a perceived level of cooperation index for each member (how much other group members perceived that particular member to have contributed). A group-level measure of perceived cooperation was then constructed by averaging the perceived level of cooperation indices for each group member. Scores for Weeks 2 through 4 were averaged to 
form an index of early perceptions of cooperation for each group, and Weeks 5 through 7 were averaged to create an index of late perceptions of cooperation for each group. Internal consistency across the four scales as indicated by the alpha for standardized variables ranged from .91 for the average of Weeks 2 through 4 to .92 for the average of Weeks 5 to 7 questionnaires.

Other dependent measures. Other measures included a measure of group identity and the group essay scores. The highest possible score on the group essay was 10 . The construction of the group identity measure is described next.

The group identity measure was constructed from eight items on the posttask questionnaire. All participants used a 7-point scale to rate the following: how happy they were feeling during that session, how pleased they were feeling during that session, how the group's interaction that day affected task performance, how the group's interaction that day affected morale, how the group's interaction that day affected interpersonal relations, what impact each member had on group cohesiveness and morale, how positive they were feeling about their group on that day, and how friendly they were feeling toward other group members.

Each student's ratings on these items were averaged to form a measure of group identity at the individual level. Member scores were averaged to yield a collective group identity score. Internal consistency of this post hoc scale (as indicated by the standardized item alpha) ranged from .91 during Weeks 2 through 4 to .86 during Weeks 5 through 7 questionnaires. A separate study that included both the items from this post hoc scale and the Hinkle Group Identity Scale (Hinkle et al., 1989) found a .74 correlation between the two scales (Arrow, 1996). In addition, because the Hinkle scale factors into three subscales measuring emotional aspects of membership (EMOT), tensions between individual needs and group dynamics (IND/GP), and cognitive aspects of identification (COG), it was possible to examine correlations between the post hoc scale and the Hinkle subscales. All correlations between the post hoc scale and the subscales were positive and statistically significant. The strongest correlation was with the EMOT subscale 
$(r=.83, p<.01)$, and the weakest correlation was with the IND/GP subscale $(r=.51, p<.01)$. The correlation with the COG subscale was .61. This pattern of correlations suggests that the post hoc scale used in both Arrow (1996) and the present study is a reasonably valid measure of group identity. ${ }^{1}$

\section{RESULTS}

All analyses were conducted at the group level. A 2 (FTF vs. $\mathrm{CMC}) \times 2$ (early vs. late) repeated measures ANOVA was conducted on the group identity measure. Table 1 shows the means by communication medium for early and late group identity scores. No significant time or medium main effects or any interactions were observed. Thus, for these groups, group identity did not change noticeably over time or with the use of different communication media. ${ }^{2}$

A 2 (FTF vs. CMC) $\times 2$ (early vs. late) repeated measures ANOVA was conducted on perceived cooperation. A significant time effect was obtained, $F(1,29)=5.73, p<.05$, with means indicating perceived cooperation increased over time (see Table 1). Medium did not exert any significant main effects or interactions. Regardless of medium, perceived cooperation increased over time in these groups.

An initial inspection of the perceived cooperation means for FTF versus CMC groups across the early and late measurements may lead one to conclude that the differences in perceived cooperation over time are minimal, suggesting that the significant main effect described earlier may be spurious. However, post hoc dependent samples $t$ tests conducted separately for the FTF and CMC groups indicated that the increase over time was significant at least for the CMC groups $(t=2.45, p<.05)$ although not for the FTF groups $(p>.05)$. That the $\mathrm{CMC}$ groups experienced more change over time than their FTF counterparts is not surprising because CMC groups studied in past research have taken longer to adjust to their novel communication technology but eventually behave similarly to their FTF counterparts on later performance measures (Hollingshead, 
TABLE 1: Early and Late Perceived Cooperation and Group Identity by Communication Medium

\begin{tabular}{|c|c|c|c|c|c|c|c|c|}
\hline & \multicolumn{4}{|c|}{ Early } & \multicolumn{4}{|c|}{ Late } \\
\hline & $F T F$ & SD & $C M C$ & SD & $F T F$ & SD & $C M C$ & SD \\
\hline Perceived cooperation & 5.16 & .60 & 5.01 & .63 & 5.25 & .58 & 5.34 & .47 \\
\hline Group identity & 5.15 & .56 & 4.87 & .57 & 4.92 & .44 & 4.85 & .55 \\
\hline
\end{tabular}

McGrath, \& O'Connor, 1994). The difference over time between the CMC and FTF groups combined was not strong enough to achieve global significance in the interaction term of the repeated measures ANOVA, however.

To further investigate what might account for the significant perceived cooperation main effect for time, a multiple regression analysis similar to the one in Bouas and Komorita (1996) was performed. Three possible predictors of perceived cooperation in the later time period were examined. First, early levels of group identity might predict later perceived cooperation. Although the analyses reported earlier suggest that this is probably not the case, the regression analysis provides a better test of this possibility given the continuous nature of the group identity and perception of cooperation measures. Thus, early group identity was included as a predictor.

A second predictor was early level of perceived cooperation. Early levels of perceived cooperation are analogous to measures of expectation of cooperation in previous research (Bouas \& Komorita, 1996). Measures of expectation of cooperation in previous research asked participants, "How many others do you think chose J [i.e., cooperated]?" and "How many others do you think chose P [i.e., defected]?" after participants had already made an anonymous choice (but before they knew what other members' choices were) in a one-shot public goods dilemma. Measures of perceived cooperation in the present study asked participants to evaluate contribution levels of group members after the more realistic public goods dilemma (i.e., group essay) was completed for that week. Previous research has shown that expectation of cooper- 
TABLE 2: Regression Weights and Change in $R^{2}$

\begin{tabular}{lccc}
\hline Measures & Full Model Weights & $\mathrm{R}^{2}$ & $\mathrm{~F}(1,27)$ for $\Delta \mathrm{R}^{2}$ \\
\hline Intercept & $2.88^{*}$ & & \\
Early group identity & -.03 & .0009 & 0.04 \\
Early perceived cooperation & $.55^{*}$ & .2803 & $12.37^{*}$ \\
Early group essay & -.03 & .0034 & 0.15 \\
\hline
\end{tabular}

NOTE: $R^{2}$ for full model $=.3881, F(1,27)=5.71, p<.005$.

$* p<.005$.

ation arising from commitments made during discussion significantly predicts future cooperation (Bouas \& Komorita, 1996). Thus, early perceived cooperation was included as a possible predictor of late perceived cooperation.

A third predictor included in the regression analysis was early performance on the group essay. Allison and Kerr (1994) found that successful groups are judged as more cooperative than unsuccessful groups and that past group success tends to engender cooperation in subsequent public goods dilemmas. If early performance on the group essay can be construed as a measure of group success, early essay performance might predict later ratings of perceived cooperation.

When early measures of group identity, perceived cooperation, and essay performance were regressed onto late measures of perceived cooperation, a significant $R^{2}$ of .39 was obtained (see Table 2). Because a significant correlation existed between two of the predictor variables (see Table 3), the beta weights in the regression equation were not interpretable. To understand the independent contribution of each predictor variable, the change in $R^{2}$ in going from the full model (all three predictors included) to a restricted model (only two predictors included) was tested for all three possible pairings of predictors. Table 2 shows that a significant change in $R^{2}$ occurred when early perceived cooperation was dropped from the equation. When early group identity and early essay performance were dropped from the equation, however, no significant change in $R^{2}$ was observed. These analyses suggest early perceived cooperation is the most important predictor of late perceived cooperation and 
TABLE 3: Intercorrelations Between Predictor Variables

\begin{tabular}{lccc}
\hline & $\begin{array}{c}\text { Early Group } \\
\text { Identity }\end{array}$ & $\begin{array}{c}\text { Early Perceived } \\
\text { Cooperation }\end{array}$ & $\begin{array}{c}\text { Early Group } \\
\text { Essay }\end{array}$ \\
\hline Early group identity & 1.00 & $.54^{*}$ & -.02 \\
Early perceived cooperation & & 1.00 & .15 \\
Early group essay & & & 1.00 \\
\hline$* p .005$. & &
\end{tabular}

fail to provide evidence that group identity plays a critical role in eliciting cooperation.

\section{DISCUSSION}

The major contribution of this study is to extend the examination of social dilemmas into a more real-life public goods setting. Besides permitting replication and generalization of prior laboratory findings, the real-life setting also permitted a more powerful test of group identity effects. Four major differences between laboratory and real-life social dilemmas were outlined: (a) Incentives may be weaker in laboratory versus real-life dilemmas; (b) communication is more constrained in the laboratory; (c) time in the laboratory refers to trial blocks within an experimental hour rather than to more extended time periods experienced by real groups; and (d) cooperation is objective in the laboratory but more subjective in real-life social dilemmas. The present study was a public goods dilemma that was more real life in terms of these four distinctions. Participants were very motivated to attain good grades on group essays, were able to communicate freely about any subject they chose, met with their group members for 2 hours each week for 7 weeks, and reported perceptions of cooperation because no objective measure of cooperation was available.

These differences have important implications for the generality of previous research on communication in social dilemmas. Previous work on communication suggests discussion of the dilemma will increase cooperation, and commitment or expectation of coop- 
eration is the critical mechanism underlying the discussion effect. Most studies investigating this problem have been conducted in controlled laboratory settings with ad hoc groups during the experimental hour. Although these studies have contributed to our understanding of cooperation in social dilemmas by providing clear, precise results, little is known about their generality. The present study extends the generality of those findings.

The differences between laboratory and real-life social dilemmas raised the possibility that group identity might account for communication's effects in more real-life social dilemma contexts than in laboratory settings. If this were the case, then group identity should increase over time and perceptions of cooperation should follow that same pattern. In fact, perceptions of cooperation did significantly increase over time in these groups, contrary to laboratory findings about cooperation's development (Isaac \& Walker, 1988; Komorita et al., 1992; Rapoport \& Chammah, 1965). Yet, group identity did not follow this same pattern. Rather, group identity showed a nonsignificant decreasing trend. Regression analyses indicated that group identity plays an insignificant role in predicting later perceived cooperation. These results support the existing body of social dilemma literature on the discussion effect in the laboratory and provide additional evidence that group identity is not an underlying mechanism of discussion's effectiveness.

Perceptions of cooperation served as a subjective cooperation measure in this study because an objective measure was unavailable. Early perceptions of cooperation were the only significant predictor of later perceptions of cooperation. Early perceptions of cooperation are analogous to measures of expectation of cooperation in previous laboratory studies of the discussion effect (Bouas \& Komorita, 1996). The early perception of cooperation measures in the present study asked participants to evaluate their own and their group members' contributions to the group essay. These measures were taken after the essay was written each week but obviously prior to the next week's essay task. Observing one's group members' contributions during one week could clearly create an expectation for what would happen the following week. 
Bouas and Komorita (1996) found that expectations of cooperation brought about by commitments made during discussion were the best predictor of objectively measured cooperation in a laboratory public goods paradigm. The present study found that early perceived cooperation was the best predictor of later perceived cooperation. Given the conceptual correspondence between early perceptions of cooperation and expectation of cooperation, the present findings in the more real-life social dilemma are entirely consistent with previous laboratory results suggesting commitments that give rise to expectations of cooperation are the more important mechanism underlying cooperation, indicating their generality.

At least two results in the present study, however, are surprising in light of existing literature. The first surprise was the lack of a relation between early performance on group essay and later perceptions of cooperation, as indicated by the regression analyses. Allison and Kerr (1994) found that successful groups are perceived to be more cooperative than unsuccessful groups and that past group success fosters cooperation in subsequent public goods. Yet, no relationship between early group performance on essays and later perceived cooperation was found in the present study. One possible explanation is that the variance on the early group performance measure $\left(\sigma^{2}=1.07\right)$ was too low to detect an existing relationship. Future research should investigate this discrepancy further.

The second surprising result was that perceived cooperation increased over time, especially in the CMC groups. Laboratory research conducted within an experimental hour suggests that cooperation should decrease over time (Komorita et al., 1992). Whereas perceived cooperation served as a subjective cooperation measure in the present study, it may be that it does not measure the same thing as objective cooperation. Future research should examine the correspondence between perceived cooperation and more objective measures of cooperation. Because some real-life social dilemmas may not have any objective means of assessing coopera- 
tion, this correspondence may be best established in laboratory conditions.

Although the present study did not entirely remove the social dilemmas from the laboratory, it meaningfully moved in that direction. With respect to the four major differences between laboratory and real-life social dilemmas outlined, the present study fell more on the side of real-life social dilemma experiences. Future work on social dilemmas occurring in less controlled situations should extend the present by engaging in explicit hypothesis testing based on the present results. If general principles of cooperation in social dilemma settings are to be identified, research must expand beyond the boundaries of the laboratory to include more realistic contexts. Doing so will not take away from laboratory findings but will strengthen those results by providing even stronger evidence of their validity and generalizability.

\section{NOTES}

1. Some readers may be interested in the relationships among items on the group identity scale used here. In the present study, when the post hoc group identity scale was subjected to a principal components factor analysis using an oblique rotation, a similar factor structure emerged across the weeks relevant to the study (Weeks 2 through 7). All weeks except for Week 5 showed that a two-factor solution accounted for anywhere between 66\% (Week 3) and $79 \%$ (Week 4) of the variance. Six of the eight items on the scale loaded on the first factor. The only items that loaded on the second factor were measures of how happy and how pleased participants indicated they were feeling during the session. These were the only two items on the scale that did not explicitly refer to feelings toward the group or its members. The most typical correlation between these two factors was approximately .44 to .51 , although during Week 6, the correlation was as low as .20.

2. An analysis was conducted on this same measure and same data in Bouas and Arrow (1996) that is similar enough to warrant explanation to avoid any confusion. Bouas and Arrow conducted a 2 (face-to-face vs. computer-mediated communication) $\times 7$ (Weeks 1 through 7) repeated measures ANOVA on the same measure of group identity in these groups as part of a larger study of the developmental pattern of group identity. The analysis reported here is conceptually similar except that rather than analyzing the time factor at seven different levels (week), this analysis did not include Week 1 and collapsed Weeks 2 through 4 into an early measure of group identity and Weeks 5 through 7 into a late measure of group identity. 


\section{REFERENCES}

Allison, S. T., \& Kerr, N. L. (1994). Group correspondence bias and the provision of public goods. Journal of Personality and Social Psychology, 66, 688-698.

Arrow, H. (1996). The development and transmission of group culture in growing organizations founded by men and women. Unpublished doctoral dissertation, University of Illinois, Urbana-Champaign.

Bouas, K. S., \& Arrow, H. (1996). The development of group identity in computer and face-to-face groups with membership change. Computer Supported Cooperative Work, 4, 153-178.

Bouas, K. S., \& Komorita, S. S. (1996). Group discussion and cooperation. Personality and Social Psychology Bulletin, 22, 1144-1150.

Braver, S. L., \& Wilson, L. A. (1986). Choices in social dilemmas: Effects of communication within subgroups. Journal of Conflict Resolution, 30, 51-62.

Brewer, M. B. (1979). In-group bias in the minimal intergroup situation: A cognitivemotivational analysis. Psychological Bulletin, 86, 307-324.

Brewer, M. B., \& Kramer, R. M. (1986). Choice behavior in social dilemmas: Effects of social identity, group size, and decision framing. Journal of Personality and Social Psychology, 50, 543-549.

Chen, X. P. (1996). The group-based binding pledge as a solution to public goods problems. Organizational Behavior and Human Decision Processes, 66, 192-202.

Chen, X. P., \& Komorita, S. S. (1994). The effects of communication and commitment in a public goods dilemma. Organizational Behavior and Human Decision Processes, 60, 367-386.

Daft, R. L., \& Lengel, R. H. (1986). Organizational information requirements, media richness, and structural design. Management Science, 32, 554-571.

Dawes, R. M. (1980). Social dilemmas. Annual Review of Psychology, 31, 169-193.

Dawes, R. M., McTavish, J., \& Shaklee, H. (1977). Behavior, communication, and assumptions about other people's behavior in a commons dilemma situation. Journal of Personality and Social Psychology, 35, 1-11.

Hill, W. F., \& Gruner, L. (1973). A study of development in open and closed groups. Small Group Behavior, 4, 355-381.

Hinkle, S., Taylor, L. A., Fox-Cardamone, D. L., \& Crook, K. (1989). Intragroup identification and intergroup differentiation: A multicomponent approach. British Journal of Social Psychology, 28, 305-317.

Hogg, M. A., \& Abrams, D. (1988). Social identification: A social psychology of intergroup relations and group processes. New York: Routledge.

Hollingshead, A. B., McGrath, J. E., \& O'Connor, K. M. (1994). Group task performance and communication technology: A longitudinal study of computer-mediated versus face-to-face work groups. Small Group Research, 24, 307-333.

Isaac, R. M., \& Walker, J. M. (1988). Group size effects in public goods provision: The voluntary contributions mechanism. Quarterly Journal of Economics, 103, 179-199.

Kelly, C. (1988). Intergroup differentiation in a political context. British Journal of Social Psychology, 27, 319-332. 
Kerr, N. L., Garst, J., Lewandowski, D. A., \& Harris, S. E. (1997). That still, small voice: Commitment to cooperate as an internalized versus a social norm. Personality and Social Psychology Bulletin, 23, 1300-1311.

Kerr, N. L., \& Kaufman-Gilliland, C. (1994). Communication, commitment, and cooperation in social dilemmas. Journal of Personality and Social Psychology, 66, 513-529.

Kiesler, S., Sproull, L., \& Waters, K. (1996). A prisoner's dilemma experiment on cooperation with people and human-like computers. Journal of Personality and Social Psychology, 70, 47-65.

Komorita, S. S., \& Parks, C. D. (1995). Interpersonal relations: Mixed-motive interaction. Annual Review of Psychology, 46, 183-207.

Komorita, S. S., Parks, C. D., \& Hulbert, L. (1992). Reciprocity and the induction of cooperation in social dilemmas. Journal of Personality and Social Psychology, 62, 607-617.

Kramer, R. M., \& Brewer, M. B. (1984). Effects of group identity on resource use in a simulated commons dilemma. Journal of Personality and Social Psychology, 46, 1044-1057.

Maples, M. F. (1988). Group development: Extending Tuckman's theory. Journal for Specialists in Group Work, 13, 17-23.

McGrath, J. E., \& Arrow, H. (1996). Introduction: The JEMCO-2 study of time, technology, and groups. Computer Supported Cooperative Work, 6, 107-126.

Messick, D. M., \& Brewer, M. B. (1983). Solving social dilemmas. In L. Wheeler \& P. Shaver (Eds.), Review of personality and social psychology (Vol. 4, pp. 11-44). Beverly Hills, CA: Sage.

O'Connor, K. M., Gruenfeld, D. H., \& McGrath, J. E. (1993). The experience of conflict in continuing work groups. Small Group Research, 24, 362-382.

Orbell, J. M., van de Kragt, A., \& Dawes, R. M. (1988). Explaining discussion-induced cooperation. Journal of Personality and Social Psychology, 54, 811-819.

Ostrom, E., \& Walker, J. M. (1991). Communication in a commons: Cooperation without external reinforcement. In T. R. Palfrey (Ed.), Laboratory research in political economy (pp. 287-322). Ann Arbor: University of Michigan Press.

Rabbie, J. M. (1982). The effects of intergroup competition and cooperation on intragroup and intergroup relationships. In V. J. Derlega \& J. Grzelak (Eds.), Cooperation and helping behavior: Theories and research (pp. 128-151). San Diego: Academic Press.

Rapoport, A., \& Chammah, A. (1965). Prisoner's dilemma. Ann Arbor: University of Michigan Press.

Tajfel, H., \& Turner, J. C. (1979). An integrative theory of intergroup conflict. In W. G. Austin \& S. Worchel (Eds.), The social psychology of intergroup relations (pp. 33-47). Pacific Grove, CA: Brooks/Cole.

Tuckman, B. W., \& Jensen, M. C. (1977). Stages of small-group development revisited. Group and Organizational Studies, 2, 419-427.

Turner, J. C. (1985). Social categorization and the self-concept: A social cognitive theory of group behavior. In E. Lawler (Ed.), Advances in group processes (Vol. 2, pp. 77-122). Greenwich, CT: JAI.

van de Kragt, A.J.C., Orbell, J. M., \& Dawes, R. M. (1983). The minimal contributing set as a solution to public goods problems. American Political Science Review, 77, 112-122.

van Lange, P.A.M., Liebrand, W.B.G., Messick, D. M., \& Wilke, H.A.M. (1992). Introduction and literature review. In W. Liebrand, D. Messick, \& H. Wilke (Eds.), Social dilemmas: Theoretical issues and research findings (pp. 3-28). New York: Pergamon. 


\section{Henry / PERCEPTIONS OF COOPERATION 527}

Kelly Bouas Henry joined the psychology faculty of Missouri Western State College in 1999. She received her doctorate in social and organizational psychology from University of Illinois, Urbana-Champaign, in 1997 and was a member of the psychology faculty at the University of Oklahoma from 1997 to 1999 . Her research interests include the antecedents and consequences of group identification, various aspects of group structure, and the development of cooperation in social dilemmas. 